Journal of Antimicrobial Chemotherapy (1986) 17, Suppl. B, 29-39

\title{
In-vitro activity of newer quinolones against aerobic bacteria
}

\author{
R. Auckenthaler, M. Michéa-Hamzehpour and J. C. Pechère \\ Laboratoire Central de Bactériologie, Hôpital Cantonal Universitaire, \\ 1211-Genève 4, Switzerland
}

\begin{abstract}
Nalidixic and five newer 4-quinolones, ciprofloxacin, enoxacin, norfloxacin, ofloxacin and pefloxacin were tested against 576 recent clinical aerobic bacterial isolates. The 4-quinolones were regularly active $\left(\mathrm{MIC}_{90}<4 \mathrm{mg} / \mathrm{l}\right)$ against the following bacteria: Staphylococcus aureus, S. epidermidis, S. saprophyticus, different Enterobacteriaceae, Haemophilus influenzae, Campylobacter jejuni, Pseudomonas aeruginosa, Agrobacter spp., Aeromonas spp., Plesiomonas spp., Neisseria meningitidis. Other bacteria were usually intermediately susceptible or resistant: different streptococci, Listeria monocytogenes, Nocardia asteroides, $P$. maltophilia, Achromobacter xylosoxydans and Alcaligenes denitrificans. Ciprofloxacin was the most potent compound, followed by ofloxacin and pefloxacin, norfloxacin and enoxacin being less active. All the 4-quinolones were much more active than nalidixic acid. The MBC/MIC ratios of the 4-quinolones were between 1 and 2 with a majority of strains, and between 2 and 3 with Streptococcus agalactiae, Str. faecalis and L. monocytogenes. A two- to eight-fold increase of MIC was observed by increasing the inoculum 10,000 -fold with most of the strains tested. Susceptible bacterial population of Klebsiella pneumoniae, Enterobacter cloacae, Serratia marcescens and $P$. aeruginosa contained more clones resistant to nalidixic acid $\left(10^{4}\right.$ to $10^{8}$ at four times the MIC) than to 4-quinolones $\left(10^{5}\right.$ to $10^{9}$ at four times the MIC). Supplementing the media with $\mathrm{MgSO}_{4}$ produced smaller inhibition zone diameters with a disc diffusion method than those obtained with non-supplemented agar, with all quinolone or strains. Less regular effect, or no effect was obtained after supplementation with $\mathrm{ZnSO}_{4}$ or $\mathrm{Ca}\left(\mathrm{NO}_{3}\right)_{2}$.
\end{abstract}

\section{Introduction}

In contrast to nalidixic acid and early derivatives such as cinoxacin, rosoxacin or flumequine, the activity of newer 4-quinolone compounds does not limit them to the oral therapy of urinary tract infections caused by Enterobacteriaceae. Recently developed 4-quinolone are characterized by a wider spectrum including Pseudomonas spp., Legionella spp. (Greenwood \& Laverick, 1983), Gram-positive bacteria and obligate intracellular organisms such as Mycobacterium spp. (Gay, DeYoung \& Roberts, 1984) or Chlamydia spp. (Heesen \& Muytiens, 1984; von Roosbroeck, Privinciael \& Caekenberghe, 1984) and Mycoplasma spp. (Ridgway et al., 1984). In contrast, the activity against anaerobic bacteria is limited. The extremely low MICs of 4-quinolones against aerobic bacteria and their pharmacokinetic properties suggests their use in numerous clinical situations. In the present study we compare the in-vitro activity of ciprofloxacin, enoxacin, norfloxacin, ofloxacin and pefloxacin against routine clinical isolates of aerobic Gram-positive and Gram-negative organisms. 


\section{Material and methods}

Antimicrobial agents. Standard powder of the following drugs were obtained from their manufacturers: Nalidixic acid (Winthrop, Switzerland), ciprofloxacin (Bayer AG, Germany), enoxacin (Roger Bellon, France), norfloxacin (Merck Sharp and Dohme, Switzerland), ofloxacin (Roussel-Hoechst, France) and pefloxacin (Rhone Poulenc, France). Antibiotic solutions were prepared in water or in broth and used immediately.

Bacterial strains. Five hundred and seventy-six clinical strains collected from patients hospitalized in the University Hospital of Geneva or, occasionally, in other Swiss hospitals, and seven ATCC control organisms were used. They were kept frozen in skim milk at $-70^{\circ} \mathrm{C}$. Before study, organisms were thawed, streaked onto sheep blood agar and incubated overnight at $35^{\circ} \mathrm{C}$.

Antimicrobial susceptibility tests. Antimicrobial activity was measured by the microdilution method in Mueller-Hinton broth (NCCLS, 1983). Disposable material was used exclusively in order to avoid cross-contamination with quinolones. The final inoculum of $10^{3}-10^{6} \mathrm{cfu} / \mathrm{ml}$ was prepared from a trypticase soy broth inoculated $4 \mathrm{~h}$ before and controlled by counting the bacteria in a calibrated volume. The inoculum for Haemophilus influenzae was prepared and tested in Brain Heart Infusion broth supplemented with $5 \%$ nicotinamide diphosphate and $10 \%$ haemin. The minimal inhibitory concentration (MIC) was read after $18 \mathrm{~h}$ of incubation at $35^{\circ} \mathrm{C}$ in air or at $42^{\circ} \mathrm{C}$ in $10 \% \mathrm{CO}_{2}$ atmosphere for Campylobacter jejuni. The minimal bactericidal concentration (MBC) was determined by subculturing $0.01 \mathrm{ml}$ on Mueller-Hinton agar and defined as $99.9 \%$ reduction in the initial inoculum. Nocardia asteroides was tested by agar dilution on Mueller-Hinton agar with $10^{4} \mathrm{cfu} / \mathrm{spot}$ with $35^{\circ} \mathrm{C}$ incubation for three days.

Effect of calcium and magnesium. Defined medium (Iso-sensitest agar, Oxoid) containing $0.3 \mathrm{mmol} \mathrm{Ca}{ }^{2+}, 1.2 \mathrm{mmol} \mathrm{Mg}^{2+}$ and $0.014 \mathrm{mmol} \mathrm{Zn}^{2+}$ respectively was supplemented in order to obtain a final concentration of 0.8 or $2.8 \mathrm{mmol} / \mathrm{Ca}(\mathrm{NO} 3)_{2}$, 2.8 or $5.0 \mathrm{mmol} / 1 \mathrm{MgSO}_{4}$, and 0.03 or $0.07 \mathrm{mmol} / 1 \mathrm{ZnSO}_{4}$.

Inoculum effect. This effect was measured on Mueller-Hinton agar with $10^{8}$ or $10^{4} \mathrm{cfu}$ per inoculum.

\section{Results}

\section{MICs of quinolones}

Against Gram-positive genera, nalidixic acid had very poor activity as shown in Table I. In contrast 96 out of 97 staphylococci were susceptible to 4-quinolones; the exception was one methicillin resistant Staphylococcus aureus which was resistant to the quinolones tested. Activity of quinolones was similar against $S$. epidermidis and three groups of $S$. aureus classified according to their susceptibility to penicillin $G$ and methicillin. S. saprophyticus was found to be one- or two-fold less susceptible than S. epidermidis. Streptococci were far less susceptible than staphylococci, most of the strains being in the intermediate susceptibility range. MICs of quinolones were similar against Streptococcus pneumoniae, Str. agalactiae and Str. faecalis. The majority of Listeria monocytogenes and $N$. asteroides were resistant to all quinolones tested except for a few sensitive strains. Quinolones were more active against the Enterobacteriaceae (Table II) than against Gram-positive bacteria. Most strains being inhibited by 
Table I. Activity of quinolones against Gram positive bacteria

\begin{tabular}{|c|c|c|c|c|}
\hline $\begin{array}{l}\text { Organisms } \\
\text { (number) }\end{array}$ & Antibiotic & $\begin{array}{l}\mathrm{MIC}_{s 0} \\
(\mathrm{mg} / \mathrm{l})\end{array}$ & $\begin{array}{l}\text { MIC }_{90} \\
(\mathrm{mg} / \mathrm{l})\end{array}$ & $\begin{array}{l}\text { Range } \\
(\mathrm{mg} / \mathrm{l})\end{array}$ \\
\hline $\begin{array}{l}\text { Penicillin-sensitive } \\
\text { S. aureus }(20)\end{array}$ & $\begin{array}{l}\text { nalidixic acid } \\
\text { ciprofloxacin } \\
\text { enoxacin } \\
\text { norfloxacin } \\
\text { ofloxacin } \\
\text { pefloxacin }\end{array}$ & $\begin{array}{l}64 \\
0-25 \\
1 \\
1 \\
0.25 \\
0.5\end{array}$ & $\begin{array}{c}>128 \\
0 \cdot 5 \\
4 \\
2 \\
1 \\
1\end{array}$ & $\begin{array}{l}32->128 \\
0.125-0.5 \\
0.5-4 \\
0.5-2 \\
0.125-1 \\
0.25-4\end{array}$ \\
\hline $\begin{array}{l}\text { Penicillin-resistant } \\
\text { Oxacillin-sensitive } \\
\text { S. aureus (19) }\end{array}$ & $\begin{array}{l}\text { nalidixic acid } \\
\text { ciprofloxacin } \\
\text { enoxacin } \\
\text { norfloxacin } \\
\text { ofloxacin } \\
\text { pefloxacin }\end{array}$ & $\begin{array}{l}64 \\
0.25 \\
1 \\
2 \\
0.25 \\
0.5\end{array}$ & $\begin{array}{l}128 \\
0.5 \\
2 \\
2 \\
0.5 \\
0.5\end{array}$ & $\begin{array}{l}32-128 \\
0.125-1 \\
0.5-4 \\
0 \cdot 5-4 \\
0.125-0.5 \\
0.25-1\end{array}$ \\
\hline $\begin{array}{l}\text { Oxacillin-resistant } \\
\text { S. aureus (24) }\end{array}$ & $\begin{array}{l}\text { nalidixic acid } \\
\text { ciprofloxacin } \\
\text { enoxacin } \\
\text { norfloxacin } \\
\text { ofloxacin } \\
\text { pefloxacin }\end{array}$ & $\begin{array}{l}64 \\
0.25 \\
1 \\
1 \\
0.25 \\
0.5\end{array}$ & $\begin{array}{l}128 \\
0-5 \\
2 \\
2 \\
0.5 \\
1\end{array}$ & $\begin{array}{l}32->128 \\
0.125-4 \\
0.5-32 \\
0.5->32 \\
0.125-2 \\
0.25-8\end{array}$ \\
\hline S. epidermidis (22) & $\begin{array}{l}\text { nalidixic acid } \\
\text { ciprofloxacin } \\
\text { enoxacin } \\
\text { norfloxacin } \\
\text { ofloxacin } \\
\text { pefloxacin }\end{array}$ & $\begin{array}{l}64 \\
0.125 \\
1 \\
0 \cdot 5 \\
0.125 \\
0.5\end{array}$ & $\begin{array}{l}128 \\
0 \cdot 25 \\
1 \\
2 \\
0 \cdot 25 \\
1\end{array}$ & $\begin{array}{l}64->128 \\
0 \cdot 125-0.25 \\
0.5-2 \\
0.5-2 \\
0.125-1 \\
0.5-2\end{array}$ \\
\hline S. saprophyticus (12) & $\begin{array}{l}\text { nalidixic acid } \\
\text { ciprofloxacin } \\
\text { enoxacin } \\
\text { norfloxacin } \\
\text { ofloxacin } \\
\text { pefloxacin }\end{array}$ & $\begin{array}{l}>128 \\
0 \cdot 25 \\
2 \\
2 \\
0 \cdot 5 \\
2\end{array}$ & $\begin{array}{c}>128 \\
0 \cdot 5 \\
4 \\
4 \\
1 \\
4\end{array}$ & $\begin{array}{l}128->128 \\
0.125-0.5 \\
1-4 \\
0.5-4 \\
0.25-1 \\
1-4\end{array}$ \\
\hline Str. pneumoniae (31) & $\begin{array}{l}\text { nalidixic acid } \\
\text { ciprofloxacin } \\
\text { enoxacin } \\
\text { norfloxacin } \\
\text { ofloxacin } \\
\text { pefloxacin }\end{array}$ & $\begin{array}{l}128 \\
0.5 \\
8 \\
4 \\
0.5 \\
4\end{array}$ & $\begin{array}{c}>128 \\
1 \\
16 \\
8 \\
1 \\
8\end{array}$ & $\begin{array}{l}32->128 \\
0.06-1 \\
4-16 \\
2-32 \\
0.015-1 \\
4-8\end{array}$ \\
\hline Str. agalactiae (19) & $\begin{array}{l}\text { nalidixic acid } \\
\text { ciprofloxacin } \\
\text { enoxacin } \\
\text { norfloxacin } \\
\text { ofloxacin } \\
\text { pefloxacin }\end{array}$ & $\begin{array}{c}>128 \\
0 \cdot 5 \\
32 \\
4 \\
1 \\
8\end{array}$ & $\begin{array}{c}>128 \\
0.5 \\
32 \\
16 \\
1 \\
16\end{array}$ & $\begin{array}{l}>128 \\
0.25-1 \\
8-32 \\
4-16 \\
0.25-2 \\
4-32\end{array}$ \\
\hline Str. faecalis (22) & $\begin{array}{l}\text { nalidixic acid } \\
\text { ciprofloxacin } \\
\text { enoxacin } \\
\text { norfloxacin } \\
\text { ofloxacin } \\
\text { pefloxacin }\end{array}$ & $\begin{array}{c}>128 \\
0 \cdot 5 \\
8 \\
4 \\
1 \\
4\end{array}$ & $\begin{array}{c}>128 \\
0.5 \\
8 \\
4 \\
2 \\
4\end{array}$ & $\begin{array}{l}>128 \\
0-25-0.5 \\
4-8 \\
2-4 \\
1-2 \\
2-4\end{array}$ \\
\hline
\end{tabular}


Table I-contd.

\begin{tabular}{lllll}
\hline $\begin{array}{l}\text { Organisms } \\
\text { (number) }\end{array}$ & Antibiotic & $\begin{array}{l}\mathrm{MIC}_{50} \\
(\mathrm{mg} / \mathrm{l})\end{array}$ & $\begin{array}{l}\mathrm{MIC}_{90} \\
(\mathrm{mg} / \mathrm{l})\end{array}$ & $\begin{array}{c}\text { Range } \\
(\mathrm{mg} / \mathrm{l})\end{array}$ \\
\hline L. monocytogenes (26) & nalidixic acid & $>128$ & $>128$ & $>128$ \\
& $\begin{array}{l}\text { ciprofloxacin } \\
\text { enoxacin }\end{array}$ & 0.5 & 1 & $0 \cdot 25-1$ \\
& norfloxacin & 4 & 16 & $4-16$ \\
& ofloxacin & 1 & 8 & $2-16$ \\
peffoxacin & 8 & 2 & $0 \cdot 5-2$ \\
N. asteroides (19) & nalidixic acid & $>128$ & $>128$ & $2-8$ \\
& ciprofloxacin & 4 & 8 & $64->128$ \\
& norfloxacin & 32 & 64 & $2-64-32$ \\
& ofloxacin & 4 & 16 & $0 \cdot 5-64$ \\
& pefloxacin & 32 & 64 & $2-64$ \\
\hline
\end{tabular}

Table II. Activity of quinolones against Enterobacteriaceae

\begin{tabular}{|c|c|c|c|c|}
\hline $\begin{array}{l}\text { Organisms } \\
\text { (number) }\end{array}$ & Antibiotic & $\begin{array}{l}\mathrm{MIC}_{50} \\
(\mathrm{mg} / \mathrm{l})\end{array}$ & $\begin{array}{l}\mathrm{MIC}_{90} \\
(\mathrm{mg} / \mathrm{l})\end{array}$ & $\begin{array}{l}\text { Range } \\
(\mathrm{mg} / \mathrm{l})\end{array}$ \\
\hline E. $\operatorname{coli}(30)$ & $\begin{array}{l}\text { nalidixic acid } \\
\text { pipemidic acid } \\
\text { ciprofloxacin } \\
\text { enoxacin } \\
\text { norfloxacin } \\
\text { ofloxacin } \\
\text { pefloxacin }\end{array}$ & $\begin{array}{l}4 \\
2 \\
0.015 \\
0.125 \\
0.06 \\
0.06 \\
0.5\end{array}$ & $\begin{array}{l}4 \\
2 \\
0.03 \\
1 \\
0.5 \\
0.06 \\
0.125\end{array}$ & $\begin{array}{l}2->128 \\
0.25->128 \\
0.004-1 \\
0.125-8 \\
0.06-4 \\
0.015-2 \\
0.06-8\end{array}$ \\
\hline Salmonella spp. (22) & $\begin{array}{l}\text { nalidixic acid } \\
\text { pipemidic acid } \\
\text { ciprofoxacin } \\
\text { enoxacin } \\
\text { norfloxacin } \\
\text { ofloxacin } \\
\text { pefloxacin }\end{array}$ & $\begin{array}{l}4 \\
2 \\
0.008 \\
0.25 \\
0.06 \\
0.03 \\
0.125\end{array}$ & $\begin{array}{l}8 \\
4 \\
0.015 \\
0.25 \\
0 \cdot 125 \\
0.06 \\
0.25\end{array}$ & $\begin{array}{l}2-8 \\
0.25-4 \\
0.004-0.015 \\
0.125-0.25 \\
0.06-0.125 \\
0.015-0.25 \\
0.06-0.25\end{array}$ \\
\hline Shigella spp. (26) & $\begin{array}{l}\text { nalidixic acid } \\
\text { pipemidic acid } \\
\text { ciprofloxacin } \\
\text { enoxacin } \\
\text { norfloxacin } \\
\text { ofloxacin } \\
\text { pefloxacin }\end{array}$ & $\begin{array}{l}2 \\
0.25 \\
0.008 \\
0.125 \\
0.06 \\
0.06 \\
0.125\end{array}$ & $\begin{array}{l}4 \\
0.25 \\
0.03 \\
0.125 \\
0.06 \\
0.125 \\
0.125\end{array}$ & $\begin{array}{l}2-4 \\
0.125-0.25 \\
0.004-0.06 \\
0.06-0.25 \\
0.06-0.125 \\
0.03-1 \\
0.06-0.125\end{array}$ \\
\hline$Y$. enterocolitica $(7)$ & $\begin{array}{l}\text { nalidixic acid } \\
\text { pipemidic acid } \\
\text { ciprofloxacin } \\
\text { enoxacin } \\
\text { norfloxacin } \\
\text { ofloxacin } \\
\text { pefloxacin }\end{array}$ & $\begin{array}{l}2 \\
0 \cdot 25 \\
0.015 \\
125 \\
0 \cdot 125 \\
0 \cdot 06 \\
0 \cdot 125\end{array}$ & $\begin{array}{l}4 \\
2 \\
0.03 \\
0.25 \\
0.125 \\
0.125 \\
0.25\end{array}$ & $\begin{array}{l}1-4 \\
0.25-4 \\
0.008-0.03 \\
0.125-0.25 \\
0.06-0.125 \\
0.03-0.125 \\
0.125-0.25\end{array}$ \\
\hline
\end{tabular}


Table II-contd.

\begin{tabular}{|c|c|c|c|c|}
\hline $\begin{array}{l}\text { Organisms } \\
\text { (number) }\end{array}$ & Antibiotic & $\begin{array}{l}\mathrm{MIC}_{50} \\
(\mathrm{mg} / \mathrm{l})\end{array}$ & $\begin{array}{l}\mathrm{MIC}_{90} \\
(\mathrm{mg} / \mathrm{l})\end{array}$ & $\begin{array}{l}\text { Range } \\
(\mathrm{mg} / \mathrm{l})\end{array}$ \\
\hline Klebsiella spp. (29) & $\begin{array}{l}\text { nalidixic acid } \\
\text { pipemidic acid } \\
\text { ciprofloxacin } \\
\text { enoxacin } \\
\text { norfloxacin } \\
\text { ofloxacin } \\
\text { pefloxacin }\end{array}$ & $\begin{array}{l}4 \\
0.5 \\
0.015 \\
0.25 \\
0.125 \\
0.125 \\
0.25\end{array}$ & $\begin{array}{l}8 \\
4 \\
0.06 \\
0.5 \\
1 \\
0.25 \\
0.5\end{array}$ & $\begin{array}{l}1-16 \\
0.25-16 \\
0.008-0.25 \\
0.06-1 \\
0.03-8 \\
0.015-0.5 \\
0.03-1\end{array}$ \\
\hline Enterobacter spp. (27) & $\begin{array}{l}\text { nalidixic acid } \\
\text { pipemidic acid } \\
\text { ciprofloxacin } \\
\text { enoxacin } \\
\text { norfloxacin } \\
\text { ofloxacin } \\
\text { pefloxacin }\end{array}$ & $\begin{array}{l}8 \\
2 \\
0.015 \\
0.25 \\
0.125 \\
0.125 \\
0.25\end{array}$ & $\begin{array}{l}32 \\
4 \\
0.03 \\
0.5 \\
0.5 \\
0.5 \\
1\end{array}$ & $\begin{array}{l}4-32 \\
0.25-16 \\
0.004-0.06 \\
0.125-1 \\
0.06-1 \\
0.03-0.5 \\
0.125-1\end{array}$ \\
\hline Ser. marcescens (23) & $\begin{array}{l}\text { nalidixic acid } \\
\text { pipemidic acid } \\
\text { ciprofloxacin } \\
\text { enoxacin } \\
\text { norfloxacin } \\
\text { ofloxacin } \\
\text { pefloxacin }\end{array}$ & $\begin{array}{l}4 \\
0.5 \\
0.125 \\
0.5 \\
0.25 \\
0.5 \\
0.5\end{array}$ & $\begin{aligned} & 16 \\
& 16 \\
& 1 \\
& 2 \\
& 1 \\
&>2 \\
& 1\end{aligned}$ & $\begin{array}{l}2-32 \\
0.25-32 \\
0.015->2 \\
0.125-4 \\
0.125-2 \\
0.125->2 \\
0 \cdot 125-4\end{array}$ \\
\hline Citrobacter freundii (13) & $\begin{array}{l}\text { nalidixic acid } \\
\text { pipemidic acid } \\
\text { ciprofloxacin } \\
\text { enoxacin } \\
\text { norfloxacin } \\
\text { ofloxacin } \\
\text { pefloxacin }\end{array}$ & $\begin{array}{l}8 \\
0.5 \\
0.008 \\
0.25 \\
0.25 \\
0.06 \\
0.125\end{array}$ & $\begin{array}{l}32 \\
0.5 \\
0.015 \\
0.5 \\
1 \\
0.25 \\
1\end{array}$ & $\begin{array}{l}4-64 \\
0.25-1 \\
0.004-0.5 \\
0 \cdot 125-2 \\
0 \cdot 125-1 \\
0.03-1 \\
0.06-2\end{array}$ \\
\hline Proteus spp. (30) & $\begin{array}{l}\text { nalidixic acid } \\
\text { pipemidic acid } \\
\text { ciprofloxacin } \\
\text { enoxacin } \\
\text { norfloxacin } \\
\text { ofloxacin } \\
\text { pefloxacin }\end{array}$ & $\begin{array}{l}8 \\
0.5 \\
0.015 \\
0.5 \\
0.125 \\
0.125 \\
0.25\end{array}$ & $\begin{array}{l}16 \\
4 \\
0.03 \\
0.5 \\
0.25 \\
0.25 \\
0.25\end{array}$ & $\begin{array}{l}2-32 \\
0.25-4 \\
0.008-0.25 \\
0.125-1 \\
0.03-0.5 \\
0.03-0.25 \\
0.125-0.5\end{array}$ \\
\hline Morganella morganii (16) & $\begin{array}{l}\text { nalidixic acid } \\
\text { pipemidic acid } \\
\text { ciprofloxacin } \\
\text { enoxacin } \\
\text { norfloxacin } \\
\text { ofloxacin } \\
\text { pefloxacin }\end{array}$ & $\begin{array}{l}4 \\
0.25 \\
0.015 \\
0.25 \\
0.06 \\
0.125 \\
0.25\end{array}$ & $\begin{array}{l}8 \\
0.5 \\
0.03 \\
0.5 \\
0.125 \\
0.25 \\
0.5\end{array}$ & $\begin{array}{l}2-16 \\
0.25-2 \\
0.004-0.03 \\
0.125-0.5 \\
0.03-0.125 \\
0.06-0.25 \\
0.06-0.5\end{array}$ \\
\hline Providencia rettgeri (6) & $\begin{array}{l}\text { nalidixic acid } \\
\text { pipemidic acid } \\
\text { ciprofloxacin } \\
\text { enoxacin } \\
\text { norfloxacin } \\
\text { ofloxacin } \\
\text { pefloxacin }\end{array}$ & $\begin{array}{l}4 \\
0.5 \\
0.03 \\
0.25 \\
0.125 \\
0.25 \\
0.25\end{array}$ & $\begin{array}{l}8 \\
0.5 \\
0.25 \\
0.5 \\
0.125 \\
1 \\
0.25\end{array}$ & $\begin{array}{l}2->128 \\
0.5-32 \\
0.008-2 \\
0.125-16 \\
0.06-16 \\
0.015->2 \\
0.125-16\end{array}$ \\
\hline
\end{tabular}


Table III. Activity of quinolones against other aerobic and mictoaerophilic Gram-negative bacteria

\begin{tabular}{|c|c|c|c|c|}
\hline $\begin{array}{l}\text { Organisms } \\
\text { (number) }\end{array}$ & Antibiotic & $\begin{array}{l}\mathrm{MIC}_{50} \\
(\mathrm{mg} / \mathrm{l})\end{array}$ & $\begin{array}{l}\mathrm{MIC}_{90} \\
(\mathrm{mg} / \mathrm{l})\end{array}$ & $\begin{array}{l}\text { Range } \\
(\mathrm{mg} / \mathrm{l})\end{array}$ \\
\hline H. influenzae (18) & $\begin{array}{l}\text { nalidixic acid } \\
\text { pipemidic acid } \\
\text { ciprofloxacin } \\
\text { enoxacin } \\
\text { norfloxacin } \\
\text { ofloxacin } \\
\text { pefloxacin }\end{array}$ & $\begin{array}{l}1 \\
2 \\
0-008 \\
0.125 \\
0.06 \\
0.015 \\
0.03\end{array}$ & $\begin{array}{l}1 \\
4 \\
0.008 \\
0.125 \\
0.06 \\
0.03 \\
0.03\end{array}$ & $\begin{array}{l}0.5-2 \\
2-128 \\
0.004-0.015 \\
0.06-0.5 \\
0-03-1 \\
0.015-0.5 \\
<0.015-1\end{array}$ \\
\hline C. jejuni (24) & $\begin{array}{l}\text { nalidixic acid } \\
\text { pipemidic acid } \\
\text { ciprofloxacin } \\
\text { enoxacin } \\
\text { norfloxacin } \\
\text { ofloxacin } \\
\text { pefloxacin }\end{array}$ & $\begin{array}{l}8 \\
16 \\
0 \cdot 25 \\
1 \\
1 \\
0.5 \\
0 \cdot 5\end{array}$ & $\begin{array}{l}16 \\
64 \\
1 \\
8 \\
8 \\
2 \\
2\end{array}$ & $\begin{array}{l}4-128 \\
8-128 \\
0 \cdot 25-2 \\
0 \cdot 5-8 \\
0 \cdot 5-8 \\
0 \cdot 25-2 \\
0 \cdot 25-4\end{array}$ \\
\hline$P$. aeruginosa (19) & $\begin{array}{l}\text { nalidixic acid } \\
\text { pipemidic acid } \\
\text { ciprofloxacin } \\
\text { enoxacin } \\
\text { norfloxacin } \\
\text { ofloxacin } \\
\text { pefloxacin }\end{array}$ & $\begin{array}{l}128 \\
32 \\
0-125 \\
1 \\
1 \\
2 \\
2\end{array}$ & $\begin{array}{l}128 \\
64 \\
0 \cdot 25 \\
2 \\
2 \\
2 \\
4\end{array}$ & $\begin{array}{l}64-128 \\
16-64 \\
0 \cdot 06-0-25 \\
0 \cdot 5-2 \\
0 \cdot 5-2 \\
0-06->2 \\
1-4\end{array}$ \\
\hline P. maltophilia (7) & $\begin{array}{l}\text { nalidixic acid } \\
\text { pipemidic acid } \\
\text { ciprofloxacin } \\
\text { enoxacin } \\
\text { norfloxacin } \\
\text { ofloxacin } \\
\text { pefloxacin }\end{array}$ & $\begin{aligned} & 8 \\
&> 64 \\
& 4 \\
& 8 \\
& 16 \\
&> 2 \\
& 2\end{aligned}$ & $\begin{aligned} & 16 \\
> & 64 \\
& 4 \\
& 8 \\
& 32 \\
> & 2 \\
& 4\end{aligned}$ & $\begin{aligned} & 8-16 \\
> & 64 \\
& 2-8 \\
& 4-16 \\
& 16->32 \\
> & 2 \\
& 2-4\end{aligned}$ \\
\hline $\begin{array}{l}\text { Other Pseudomonas spp. } \\
\text { (not aeruginosa) (8) }\end{array}$ & $\begin{array}{l}\text { nalidixic acid } \\
\text { pipemidic acid } \\
\text { ciprofloxacin } \\
\text { enoxacin } \\
\text { norfloxacin } \\
\text { ofloxacin } \\
\text { pefloxacin }\end{array}$ & $\begin{array}{l}4 \\
4 \\
0 \cdot 125 \\
0.5 \\
0.5 \\
0.25 \\
1\end{array}$ & $\begin{array}{l}64 \\
8 \\
2 \\
2 \\
2 \\
1 \\
2\end{array}$ & $\begin{array}{l}0.5-64 \\
2-64 \\
0.03-2 \\
0.06-4 \\
0.06-4 \\
0.06-2 \\
0.06-4\end{array}$ \\
\hline $\begin{array}{l}\text { Achromo. xylosoxydans } \\
\text { and Alcalig. } \\
\text { denitrificans (6) }\end{array}$ & $\begin{array}{l}\text { nalidixic acid } \\
\text { pipemidic acid } \\
\text { ciprofloxacin } \\
\text { enoxacin } \\
\text { norfloxacin } \\
\text { ofloxacin } \\
\text { pefloxacin }\end{array}$ & $\begin{array}{l}16 \\
128 \\
2 \\
16 \\
>32 \\
\quad 4 \\
8\end{array}$ & $\begin{aligned} & 32 \\
> & 128 \\
> & 2 \\
> & 32 \\
> & 32 \\
& 32 \\
& 16\end{aligned}$ & $\begin{aligned} & 16-32 \\
> & 128 \\
& 1->2 \\
& 16->32 \\
> & 32 \\
& 4-32 \\
& 4-16\end{aligned}$ \\
\hline Acinetobacter spp. (15) & $\begin{array}{l}\text { nalidixic acid } \\
\text { pipemidic acid } \\
\text { ciprofloxacin } \\
\text { enoxacin } \\
\text { norfloxacin } \\
\text { ofloxacin } \\
\text { pefloxacin }\end{array}$ & $\begin{array}{l}4 \\
16 \\
0.06 \\
0.5 \\
2 \\
0.25 \\
0.25\end{array}$ & $\begin{array}{l}8 \\
128 \\
0 \cdot 06 \\
4 \\
16 \\
1 \\
1\end{array}$ & $\begin{array}{l}0.5-8 \\
1-128 \\
0.004-0.125 \\
0.004-4 \\
0.03-32 \\
0.015-1 \\
0.03-4\end{array}$ \\
\hline
\end{tabular}


Table III-contd.

\begin{tabular}{|c|c|c|c|c|}
\hline $\begin{array}{l}\text { Organisms } \\
\text { (number) }\end{array}$ & Antibiotic & $\begin{array}{l}\mathrm{MIC}_{50} \\
(\mathrm{mg} / \mathrm{l})\end{array}$ & $\begin{array}{l}\mathrm{MIC}_{90} \\
(\mathrm{mg} / \mathrm{l})\end{array}$ & $\begin{array}{l}\text { Range } \\
(\mathrm{mg} / \mathrm{l})\end{array}$ \\
\hline Agrobacter spp. (20) & $\begin{array}{l}\text { nalidixic acid } \\
\text { pipemidic acid } \\
\text { ciprofloxacin } \\
\text { enoxacin } \\
\text { norfloxacin } \\
\text { ofloxacin } \\
\text { pefloxacin }\end{array}$ & $\begin{array}{l}16 \\
4 \\
0.06 \\
0.25 \\
0.5 \\
0.25 \\
0.25\end{array}$ & $\begin{array}{l}16 \\
4 \\
0.06 \\
0.5 \\
1 \\
0.5 \\
0.25\end{array}$ & $\begin{array}{l}8-32 \\
2-32 \\
0-03-0.06 \\
0.25-0.5 \\
0.125-1 \\
0.25-1 \\
0.125-0.5\end{array}$ \\
\hline $\begin{array}{l}\text { Aeromonas and } \\
\quad \text { Plesiomonas spp. (19) }\end{array}$ & $\begin{array}{l}\text { nalidixic acid } \\
\text { pipemidic acid } \\
\text { ciprofloxacin } \\
\text { enoxacin } \\
\text { norfloxacin } \\
\text { ofloxacin } \\
\text { pefloxacin }\end{array}$ & $\begin{array}{l}1 \\
0.125 \\
0.004 \\
0.06 \\
0.03 \\
0.015 \\
<0.015\end{array}$ & $\begin{array}{l}2 \\
0.25 \\
0.008 \\
0.125 \\
0.06 \\
0.015 \\
0.06\end{array}$ & $\begin{array}{c}0.5-2 \\
0.125-0.5 \\
<0-001-0.015 \\
0.03-0.125 \\
<0.015-0.06 \\
0.008-0.03 \\
<0.015-0.06\end{array}$ \\
\hline$N$. meningitidis (18) & $\begin{array}{l}\text { nalidixic acid } \\
\text { ciprofloxacin } \\
\text { enoxacin } \\
\text { norfloxacin } \\
\text { ofloxacin } \\
\text { pefloxacin }\end{array}$ & $\begin{array}{l}1 \\
0-002 \\
0-03 \\
0-03 \\
0-008 \\
0.03\end{array}$ & $\begin{array}{l}2 \\
0.004 \\
0.06 \\
0.03 \\
0.015 \\
0.03\end{array}$ & $\begin{array}{l}0.5-2 \\
0-002-0-004 \\
<0-015-0.06 \\
<0.015-0.03 \\
0.004-0.015 \\
<0-015-0.06\end{array}$ \\
\hline
\end{tabular}

$<1 \mathrm{mg} / 1$. Some genera were rather more susceptible, such as Escherichia coli, Salmonella spp., Shigella spp. and Yersinia enterocolitica, while others were distinctly more resistant, such as Enterobacter spp., Serratia marcescens and Providencia rettgeri. Although not shown in Table II, ampicillin, carbenicillin, cephalothin, cefotaxime, gentamicin and amikacin were tested simultaneously with the quinolones. Many of the strains of enterobacteria used were resistant to penicillins, cephalothin and gentamicin. No relationship between resistance to these agents and to 4-quinolones could be found. However, several strains showed a high level of resistance to nalidixic acid not seen with the 4-quinolones. As well as the Enterobacteriaceae, many other aerobic Gramnegative bacteria were susceptible to quinolones (Table III). These agents were extremely active against Aeromonas spp., Plesiomonas spp., Neisseria meningitidis and $H$. influenzae. Among the pseudomonas, P.maltophilia was more resistant than $P$. aeruginosa. The quinolones showed activity against $C$. jejuni and Agrobacter spp. comparable to that against the majority of enterobacteria, while Achromoxylosoxydans and Alcaligenes denitrificans were generally resistant. Acinetobacter spp. were susceptible to wide range concentrations of quinolones.

Comparing the activity of the different quinolones tested on a weight basis, ciprofloxacin was the most potent compound, followed by ofloxacin and pefloxacin. Norfloxacin and enoxacin were less active than pefloxacin. Differences between these compounds were more pronounced with Gram-negative bacteria than with Grampositive genera. All the 4-quinolones were more active than nalidixic acid.

$M B C s$ of quinolones. The MBC/MIC ratios of pefloxacin, norfloxacin and enoxacin were between 1 and 2 for the majority of strains, and between 2 and 3 with Str. 
Table IV. Mean MDC/MIC ratios for five quinolones

\begin{tabular}{|c|c|c|c|c|c|c|}
\hline Organisms & $\begin{array}{l}\text { Number } \\
\text { tested }\end{array}$ & $\begin{array}{l}\text { Nalidixic } \\
\text { acid }\end{array}$ & $\begin{array}{l}\text { Cipro- } \\
\text { floxacin }\end{array}$ & Enoxacin & $\begin{array}{l}\text { Nor- } \\
\text { floxacin }\end{array}$ & Pefloxacin \\
\hline $\begin{array}{l}\text { Penicillin-sensitive } \\
\text { S. aureus }\end{array}$ & 14 & $1 \cdot 7$ & 1.5 & 1.64 & 1.64 & $1 \cdot 4$ \\
\hline $\begin{array}{l}\text { Penicillin-resistant } \\
\text { Methicillin-sensitive } \\
\text { S. aureus }\end{array}$ & 11 & $2 \cdot 3$ & $2 \cdot 4$ & 1.45 & 20 & 1.9 \\
\hline $\begin{array}{l}\text { Penicillin-resistant } \\
\text { Metbicillin-resistant } \\
\text { S. aureus }\end{array}$ & 16 & $1 \cdot 7$ & $2 \cdot 7$ & 1.6 & $2 \cdot 2$ & $1 \cdot 7$ \\
\hline S. epidermidis & 17 & 1.7 & 2.9 & 1.7 & 1.9 & $1 \cdot 4$ \\
\hline S. saprophyticus & 11 & inactive & $2 \cdot 1$ & 1.6 & 1.7 & $1 \cdot 2$ \\
\hline Str. agalactiae & 14 & inactive & $2 \cdot 0$ & N.D. & $2 \cdot 6$ & $2 \cdot 5$ \\
\hline Str. faecalis & 16 & inactive & 1.6 & 3.5 & $2 \cdot 1$ & $2 \cdot 9$ \\
\hline Str. pneumoniae & 11 & inactive & $1 \cdot 3$ & $1 \cdot 3$ & $1 \cdot 3$ & $1 \cdot 2$ \\
\hline L. monocytogenes & 10 & inactive & $2 \cdot 1$ & $2 \cdot 4$ & $2 \cdot 2$ & 1.9 \\
\hline E. coli & 12 & $2 \cdot 1$ & $1 \cdot 0$ & $1 \cdot 3$ & $1 \cdot 6$ & $1 \cdot 8$ \\
\hline Shigella spp. & 24 & 1.8 & N.D. & $1 \cdot 1$ & $1 \cdot 3$ & $1 \cdot 1$ \\
\hline Salmonella spp. & 29 & $2 \cdot 2$ & $1 \cdot 0$ & 1.6 & $1 \cdot 2$ & 1.5 \\
\hline Enterobacter spp. & 13 & $1 \cdot 5$ & 10 & $1 \cdot 3$ & $1 \cdot 1$ & $1 \cdot 1$ \\
\hline Klebsiella spp. & 15 & $3 \cdot 0$ & $1 \cdot 1$ & $1 \cdot 3$ & $1 \cdot 3$ & $1 \cdot 7$ \\
\hline Serratia spp. & 18 & $3 \cdot 2$ & N.D. & $1 \cdot 8$ & $2 \cdot 1$ & $2 \cdot 1$ \\
\hline Proteus spp. & 20 & $2 \cdot 0$ & $1 \cdot 4$ & 1.6 & $1 \cdot 5$ & $1 \cdot 8$ \\
\hline P. aeruginosa & 10 & 1.7 & $4 \cdot 5$ & 1.6 & 1.8 & 1.9 \\
\hline $\begin{array}{l}\text { Pseudomonas spp. } \\
\text { (not aeruginosa) }\end{array}$ & 15 & $2 \cdot 7$ & $2 \cdot 5$ & $1 \cdot 8$ & $2 \cdot 1$ & $1 \cdot 9$ \\
\hline
\end{tabular}

* N.D. a not determined.

agalactiae, Str. faecalis and L. monocytogenes (Table IV). In comparison with these three agents, bactericidal activity of ciprofloxacin was somewhat different, with higher MBC/MIC ratios against most staphylococci and Pseudomonas, but lower ratios against Str. agalactiae, Str. faecalis, and enterobacteria.

Inoculum effect and mutational frequencies. Determinations of MICs with two inocula of $10^{4}$ and $10^{8} \mathrm{cfu}$ showed an inoculum effect particularly obvious with Enterobacter cloacae and Ser. marcescens (Table V). The inoculum effect was most

Table V. Fold increase of MICs with an inoculum of $10^{8} \mathrm{cfu}$ per spot as compared to an inoculum of $10^{4}$ cfu per spot

\begin{tabular}{|c|c|c|c|c|c|c|}
\hline Strains & $\begin{array}{l}\text { Number } \\
\text { tested }\end{array}$ & $\begin{array}{l}\text { nalidixic } \\
\text { acid }\end{array}$ & $\begin{array}{l}\text { Fold in } \\
\text { cipro- } \\
\text { floxacin }\end{array}$ & $\begin{array}{l}\text { we of MIC } \\
\text { enoxacin }\end{array}$ & $\begin{array}{c}\text { ge with: } \\
\text { nor- } \\
\text { floxacin }\end{array}$ & pefloxacin \\
\hline E. coli & 4 & $1-4$ & $2-4$ & $1-2$ & $1-2$ & 2 \\
\hline K. preumoniae & 4 & $4-8$ & $2-8$ & $1-4$ & $2-8$ & $2-8$ \\
\hline E. cloacae & 4 & $8-32$ & $1-16$ & $1-32$ & $2-8$ & $4-16$ \\
\hline Ser. marcescens & 4 & $8-16$ & $4-16$ & $8-32$ & 8 & $4-8$ \\
\hline$P$. aeruginosa & 4 & $4-16$ & $4-8$ & $2-8$ & $4-16$ & $2-8$ \\
\hline S. aureus & 4 & $2-4$ & $2-4$ & $1-4$ & $2-4$ & $2-4$ \\
\hline Str. faecalis & 4 & resistant & $2-8$ & $8-16$ & $2-4$ & $2-8$ \\
\hline
\end{tabular}


Table VI. Log values of the number of colonies growing on Mueller-Hinton Agar containing quinolone concentrations four or eight times the MICs (inoculum: $10^{9} \mathrm{cfu}$ )*

\begin{tabular}{|c|c|c|c|c|c|c|c|c|c|c|c|c|}
\hline \multirow{2}{*}{$\begin{array}{l}\text { Strains } \\
\text { (number) }\end{array}$} & \multicolumn{12}{|c|}{$\begin{array}{l}\text { Log of colonies growing at four or eight times the MIC of: } \\
\text { nalidixic cipro- }\end{array}$} \\
\hline & $\begin{array}{l}4 \times \\
\text { MIC }\end{array}$ & $\begin{array}{l}8 \times \\
\text { MIC }\end{array}$ & $\begin{array}{l}4 \times \\
\text { MIC }\end{array}$ & $\begin{array}{l}8 \times \\
\text { MIC }\end{array}$ & $\begin{array}{l}4 \times \\
\text { MIC }\end{array}$ & $\begin{array}{l}8 \times \\
\text { MIC }\end{array}$ & $\begin{array}{l}4 \times \\
\text { MIC }\end{array}$ & $\begin{array}{l}8 \times \\
\text { MIC }\end{array}$ & $\begin{array}{l}4 \times \\
\text { MIC }\end{array}$ & $\begin{array}{l}8 \times \\
\text { MIC }\end{array}$ & $\begin{array}{l}4 \times \\
\text { MIC }\end{array}$ & $\begin{array}{l}8 \times \\
\text { MIC }\end{array}$ \\
\hline E. coli (4) & 0 & 0 & 0 & 0 & 0 & 0 & 0 & 0 & 0 & 0 & 0 & 0 \\
\hline$K$. pnetimoniae (4) & $1-2$ & 0 & $0-1$ & 0 & $0-2$ & 0 & $0-2$ & $0-1$ & $0-2$ & $0-1$ & $0-2$ & 0 \\
\hline E. cloacae (4) & $1-2$ & $1-2$ & $0-2$ & $0-2$ & $0-2$ & $0-2$ & $0-2$ & $0-1$ & $0-3$ & $0-2$ & $0-2$ & $0-2$ \\
\hline Ser. marcescens (4) & $2-4$ & $1-3$ & $0-4$ & $0-2$ & $2-4$ & $0-4$ & $2-4$ & $0-1$ & $0-4$ & $0-2$ & $1-\overline{3}$ & $0-1$ \\
\hline P. aeruginosa (4) & $1-2$ & $0-2$ & $1-3$ & 0 & $1-2$ & $0-1$ & $1-2$ & $0-2$ & $0-2$ & $0-1$ & $0-2$ & $0-1$ \\
\hline$S$. aureus (4) & $3-5$ & 0 & $0-2$ & 0 & 0 & 0 & $0-1$ & $0-1$ & 0 & 0 & $0-2$ & $0-1$ \\
\hline
\end{tabular}

* No colonies grew at 16 times the MIC with either compound.

marked with nalidixic acid while the differences found between the 4-quinolones were not significant. Data of Table $\mathrm{V}$ correlated well with determination of mutation frequencies shown in Table VI. E. coli populations contained less than $10^{9}$ mutants resistant to the quinolones tested. Within the other strains studied, Ser. marcescens and $E$. cloacae had the highest frequency of mutants, and we found more clones resistant to nalidixic acid $\left(10^{4}-10^{8}\right.$ at a concentration of four times the MICs) than to 4-quinolones $\left(10^{5}-10^{9}\right.$ at four times the MICs). All the 4-quinolones tested selected resistant mutants at similar frequencies. No colonies grew at 16 times the MICs with either compounds.

Influence of cation supplementation. Addition of 0.03 or $0.06 \mathrm{mmol}$ of $\mathrm{ZnSO}_{4}$ did not alter the size of the inhibition zone diameters produced by ciprofloxacin, ofloxacin, pefloxacin and gentamicin when testing six strains in a disc-diffusion method (Figure 1). Supplementation with $\mathrm{Ca}\left(\mathrm{NO}_{3}\right)_{2}$ reduced the inhibition zone diameters of ofloxacin, pefloxacin and gentamicin but not those of ciprofloxacin. Supplementation with $\mathrm{MgSO}_{4}$ produced significantly smaller inhibition zone diameters than those obtained with non-supplemented agar in all cases, whatever the antibiotic or the strain considered.

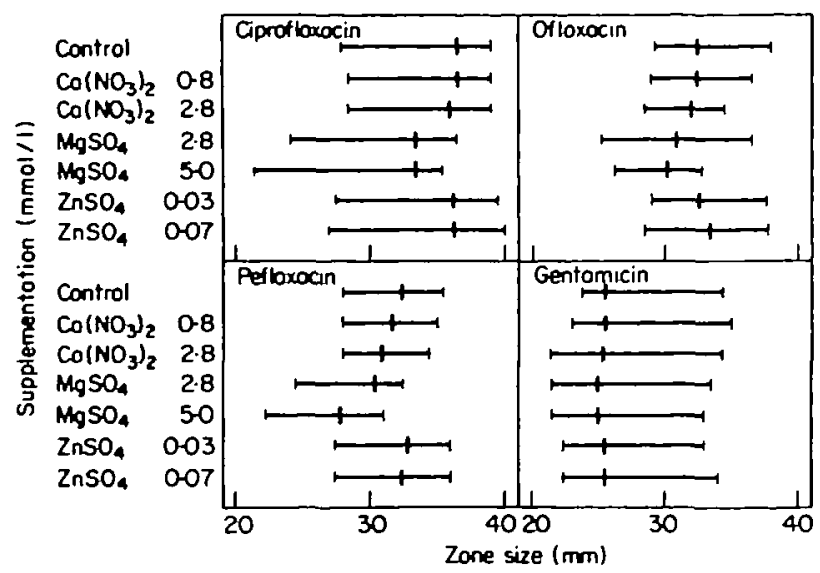

Figare 1. Influence of cation supplementation on the inhibition zone size obtained by disc diffusion with four antibiotics. 


\section{Discussion}

Newer 4-quinolones are characterized by a broader spectrum of activity when compared to nalidixic acid. The data of this study confirm the similarity of the antibacterial spectrum for the 4-quinolone compounds tested (Wise, Andrews \& Edwards, 1983; Barry et al., 1984; Bauernfeind \& Petermüller, 1984; van Caekenberghe \& Pattyn, 1984; Chin \& Neu, 1984; Hoogkamp, 1984). 4-quinolones are active against all Enterobacteriaceae, including Enterobacter spp. or Ser. marcescens which are often resistant also to newer cephalosporins. In particular organisms causing diarrhoea including Campylobacter spp. are very sensitive and may therefore be useful in this clinical situation. Against $P$. aeruginosa the activity is modest and not related to concomittant resistance to carbenicillin or gentamicin. Other non-fermenters such as $P$. maltophilia, Achromobacter spp. or Alcaligenes spp. are more than ten times less sensitive and often resistant to quinolones. The spectrum of 4-quinolones also includes Gram-positive organisms including staphylococci and to a lesser degree streptococci and Listeria spp. The MICs of Nocardia spp. are close to the achievable peak serum levels of quinolones and therefore cannot be recommended for clinical trials. As found by other authors (Bauernfeind \& Petermüller, 1984; van Caekenberghe \& Pattyn, 1984) the activity on a weight basis is highest for ciprofloxacin, followed by pefloxacin and ofloxacin, norfloxacin, enoxacin and the least active nalidixic acid. However, this observation will have to be matched with pharmacokinetics and clinical results, because other examples have taught, that in-vitro activity is not a sufficient criterion for the evaluation of new antimicrobial agents.

The bactericidal activity of quinolones is in general within 1-2-fold the MIC. Only Str. agalactiae, Str. faecalis, and L. monocytogenes have MBC two to three times higher than the MIC. Therefore the use of quinolones particularly for enterococcal endocarditis has first to be confirmed in the animal model. Ciprofloxacin has higher MBC-MIC ratios against $P$. aeruginosa when compared to the other 4-quinolones.

Emergence of resistance during therapy has been noted with nalidixic acid (Slack, 1984), and newer 4-quinolones (Acar, Kitzis \& Goldstein, 1985; Lauwers et al., 1985). In this study we have evaluated the frequency of resistant mutations by measuring the increase of MIC by raising the inoculum 10,000 fold. The increase was two- to eightfold for most of the organisms tested. However, Enterobacter spp. and Ser. marcescens both well known for emergence of resistance during therapy with cephalosporin (Sanders \& Sanders, 1985), again have a higher increase of the MIC up to 32 times and this could well explain the clinical failures. The mechanism of resistance is not known, but could be explained by a defect in bacterial penetration as the observation is common to all 4-quinolones tested (Smith, 1984).

The influence of $\mathrm{pH}$, cations and various media has been mentioned by various authors. We have measured the influence of increased calcium, magnesium or zinc concentration on the zone size of a disc diffusion test. At the tested concentrations only magnesium reduced the zone size significantly for all compounds. The mechanism of impaired activity in presence of magnesium is unknown. Possibly, magnesium might interfere at least at two levels, either on the outer membrane as is the case with aminoglycosides, or at the level of DNA-gyrase-DNA interaction. The clinical significance of the magnesium effect is unknown. However, increased magnesium concentrations in the urine, together with a low $\mathrm{pH}$, could be responsible for impaired therapeutic response in difficult to treat urinary tract infections. In addition, in testing 
susceptibility of 4-quinolones, concentration of the magnesium in the medium should be standardized.

\section{References}

Acar, J., Kitzis, M.D. \& Goldstein, F. (1985). Frequency and mutation rate of new quinolones compared to nalidixic acid. In 14th International Congress of Chemotherapy, Kyoto. Abstract, WS-7-6.

Barry, A. L., Jones, R. N., Thornsberry, C., Ayers, L. W., Gerlach, E. H. \& Sommers, H. M. (1984). Antibacterial activities of ciprofloxacin, norfloxacin, oxolinic acid, cinoxacin, and nalidixic acid. Antimicrobial Agents and Chemotherapy 25, 633-7.

Bauernfeind, A. \& Petermüller, C. (1984). Vergleich der antibacteriellen Profile von Ciprofloxacin, Norfloxacin, Pefloxacin, Ofloxacin, Enoxacin, Pipemidsäure und Nalidixinsäure. Fortschritte antimikrobieller und antineoplastischer Chemotherapie 3-5, $531-48$.

van Caekenberghe, D. L. \& Pattyn, S. R. (1984). In vitro activity of ciprofloxacin compared with those of other new fluorinated piperazinyl-substituted quinoline derivatives. Antimicrobial Agents and Chemotherapy 25, 518-21.

Chin, N. X. \& Neu, H. C. (1984). Ciprofloxacin, a quinolone carboxylic acid compound active against aerobic and anaerobic bacteria. Antimicrobial Agents and Chemotherapy 25, 319-26.

Greenwood, D. \& Laverick, A. (1983). Activities of newer quinolones against Legionella group organisms. Lancet ii, $279-80$.

Gay, D., DeYoung, D. R. \& Roberts, G. D. (1984). In vitro activities of norfloxacin and ciprofloxacin against Mycobacterium tuberculosis, $M$. avium complex, $M$. chelonei, M. fortuitum, and M. kansasii. Antimicrobial Agents and Chemotherapy 26, 94-6.

Heesen, F. W. A. \& Muytiens, H. L. (1984). In vitro activities of ciprofloxacin, norfloxacin, pipemidic acid, cinoxacin, and nalidixic acid against Chlamydia trachomatis. Antimicrobial Agents and Chemotherapy 25, 123-4.

Hoogkamp, J. A. (1984). Comparative in vitro activity of five quinoline derivatives and five other antimicrobial agents used in oral therapy. European Journal of Clinical Microbiology $3,333-8$

Lauwers, S., Vincken, W., Naessens, A. \& Pierard, D. (1985). The efficacy and safety of pefloxacin in the treatment of severe infections in patients hospitalized in intensive care units. In 14th International Congress of Chemotherapy, Kyoto. Abstract, WS-7-11.

National Committee for Clinical Laboratory Standards (1983). Methods for dilution antimicrobial susceptibility tests for bacteria that grow aerobically. Tentative Standard M7-T. National Committee for Clinical Laboratory Standards, Villanova, Pa.

Ridgway, G. L., Mumtaz, G., Gabriel, F. G. \& Oriel, D. J. (1984). The activity of ciprofloxacin and other 4-quinolones against Chlamydia trachomatis and Mycoplasma in vitro. European Journal of Clinical Microbiology 3, 344-6.

van Roosbroeck, R. J., Privinciael, D. R. \& van Caekenberghe, D. L. (1984). Activity of the newer quinolones against Chlamydia trachomatis. British Journal of Veneral Disease 60, 350.

Sanders, C. C. \& Sanders, E. Jr. (1985). Microbial resistance to newer generation betalactam antibiotics: clinical and laboratory implications. Journal of Infectious Diseases 151, 399-406.

Slack, R. (1984). Review of bacterial resistance, a challenge to the treatment of urinary infections. Journal of Antimicrobial Chemotherapy 13, Suppl. B, 1-7.

Smith, J. T. (1984). Chemistry and mode of action of 4-quinolone agents. Fortschritte antimikrobieller und antineoplastischer Chemotherapie 3-5, 493-508.

Wise, R., Andrews, J. M. \& Edwards, L. J. (1983). In vitro activity of Bay 09867, a new quinoline derivative, compared with those of other antimicrobial agents. Antimicrobial Agents and Chemotherapy 23, 559-64. 